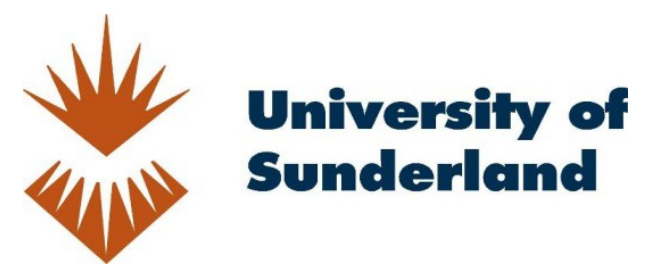

Campos, Jamie, Jantunen, Erkki, Baglee, David, Gilabert, Eduardo, Fumagali, Luca and Emmanouilidis, Christos (2016) The Use of Mobile Technologies and Their Economic Benefits in Maintenance. In: Proceedings of the 10th World Congress on Engineering Asset Management (WCEAM 2015). Springer International Publishing, Springer International Publishing Switzerland, pp. 69-71. ISBN 978-3-319-27062-3

Downloaded from: http://sure.sunderland.ac.uk/id/eprint/6081/ 
Please refer to the usage guidelines at http://sure.sunderland.ac.uk/policies.html or alternatively contact sure@sunderland.ac.uk. 


\title{
The Use of Mobile Technologies and Their Economic Benefits in Maintenance
}

\author{
Jaime Campos ${ }^{1}$, Erkki Jantunen ${ }^{2}$, David Baglee ${ }^{3}$, Eduardo Gilabert ${ }^{4}$, Luca \\ Fumagalli ${ }^{5}$, Christos Emmanouilidis 6
}

\begin{abstract}
The use of mobile devices enables maintenance staff to gain access to information and services relevant to the task in hand in near real time and wherever some form of network access is provided. Essentially, mobile device users become mobile actors who dynamically interact with the physical environment, i.e. workplace, and supporting information systems, leading to faster response to events and an increased performance. This paper provides insight into the acceptance prospects and best practices of the mobile technologies in the area of maintenance. Furthermore, the potential financial impact is discussed as a key driver for the adoption of mobile technologies in the maintenance practice.
\end{abstract}

\footnotetext{
${ }^{1}$ J.Campos $(\triangle)$

Department of Informatics, Linnaeus University, Sweden

Jaime.Campos@lnu.se

${ }^{2}$ E. Jantunen

VTT Technical Research Centre of Finland, P.O.Box 1000, FI-02044 VTT, Finland,

erkki.jantunen@vtt.fi

${ }^{3}$ D. Baglee

Department of Computing, Engineering and Technology, University of Sunderland, UK

david.baglee@sunderland.ac.uk

${ }^{4}$ E. Gilabert

Fundación TEKNIKER, Inaki Goegana 5, 20600 Eibar, Gipuzkoa, Spain

eduardo.gilabert@tekniker.es

${ }^{5}$ L. Fumagalli

Department of Management, Economics and Industrial Engineering, Politecnico di Milano, Italy

luca1.fumagalli@polimi.it

${ }^{6} \mathrm{C}$. Emmanouilidis

ATHENA Research \& Innovation Centre, Greece

christosem@ieee.org
} 
${ }^{1}$ J.Campos - Mobile Technologies Acceptance and their Economic Benefits in Maintenance

\section{Introduction}

Mobile technologies usage in maintenance can have many benefits, with increased organisational performance being a key expectation. However, mobile devices have not been exploited to their full potential in industrial daily practice by maintenance departments. It is, therefore, important to be aware of the factors that might impede the integration of the mobile technologies into the field. Thus, technology acceptance theories are becoming important contributing to better understanding. Hence, it is, crucial to understand those factors, since mobile technology has the potential to help maintenance managers automate data collection and analyses, support processes and reduce maintenance costs (Lee et al., 2004; Baglee and Knowles, 2013). Reviews in the field highlighted both the significant potential and rapid advances in the field of Information and Communication Technologies (ICTs) in Condition Monitoring and Maintenance (Campos and Prakash, 2006; Campos, 2009). Furthermore, the importance of a costeffectiveness analysis is key because it is the way to indicate if any profit or competitive advantage can be achieved by using more automatic maintenance tasks by means of ICTs, especially in predictive maintenance (Conde et al., 2009; Alrabghi and Tiwari 2013). The current paper goes through the technology acceptance theories and best practices in section 2. Section 3 deals with the economic benefits of using the mobile technologies in maintenance. Further on, in section 4 , the main statistics and lessons learned from a survey carried out on the use of mobile devices are considered. In section 5 the discussion and conclusions are presented.

\section{Technology acceptance and best practices}

The acceptance of Information Systems (IS) by users has been researched for several years and has involved cross-disciplinary domains with a specific focus on explaining the intentional variance of Information and Communication Technologies (ICTs) acceptance by users and understanding technology acceptance. These theoretical developments regarding the possible motives and barriers on the way of accepting and adopting the technologies are manifold and have resulted in several acceptance models. For instance, the Unified Theory of Acceptance and Use of Technology (UTAUT) assume four concepts affecting the behavioural intent and usage behaviour of ICTs, namely: the performance expectancy, the effort expectancy; the social influence and the facilitating conditions. However, the concern about the UTAUT model is that it does not include attitude and self-efficacy as direct determinants of behavioural intention in the UTAUT model, which have proved to be important factors with the help of the technology acceptance research (Venkatesh et al., 2003). ICTs enablers can support enterprises in meeting expected benefits such as an increased organisational performance and productivity, insofar as they are accepted and used by employees in an organization (Venkatesh 
${ }^{1}$ J.Campos - Mobile Technologies Acceptance and their Economic Benefits in Maintenance

et al., 2003). Consequently, the IT adoption technologies with their different factors are important to understand in order to be able to facilitate the acceptance of any kind of ICTs as well as the mobile technologies in the maintenance department. Apart from studies linked to technology acceptance theories, other efforts have focused more on the specific domain of maintenance and asset management. In Europe, the European Federation of National Maintenance Societies (EFNMS), through its European Asset Management Committee (EAMC) performed a survey of EU industry regarding the state of play in Asset Management best practices (EFNMS/EAMC, 2012). How organisations manage data related to their assets is of particular interest. It is worth noting that although maintenance - related events were typically recorded in systems such as Computerised Maintenance Management Systems (CMMS), the loop between acquiring information and reaching decisions is not actually closed, leaving much room for improvement regarding the usage of ICTs enablers. According to the EFNMS report, part of the problem is specifically related to the fact that quality or actionable data may not, in most cases, be available at the right time and in the right place and to the persons authorised to have access to it. This highlights the need for greater penetration and adoption of ICTs, especially mobile ICT solutions. A key area of improvement that mobile ICTs can contribute to is enabling true mobile collaboration (Emmanouilidis et al., 2009). Specifically, removing time and space constraints, mobile maintenance staff can take advantage of portability, accessibility, reachability, identification and localisation features when interacting with a maintenance - oriented information system and relevant physical environment in the workplace. Taking into account that among the main factors impeding successful CMMS implementation in industry are selection errors, insufficient commitment, poor training, limited addressing of key organisational issues, underestimation of difficulties and consequent limited allocation of resources and lack of a demonstrable use of system output, an expert opinion study has produced some interesting results regarding expectations and adoption prospects for mobile ICTs in maintenance and asset management. Upon consulting experts in the field, the study considered technological, organisational and human capital issues, relevant to mobile ICTs adoption and prospects in this domain (Sayfar and Gao, 2013). Specifically, the study suggested that in order to improve the adoption effort, it should be accompanied by relevant business process alignment in the three broad organisational layers (strategic, tactical and operational) in company's activities and should prioritise implementation, targeting the most critical processes or assets to demonstrate impact. Leaving the broader picture to focus on individual projects and specific implementation and technology adoption aspects, the results may follow not only the general pattern but can also provide additional and more focused insight that can be obtained regarding specific technological solutions. For example, the adoption of e-training solutions in maintenance and asset management depends on aspects of introduced technological elements, functional features as well as con- 
${ }^{1}$ J.Campos - Mobile Technologies Acceptance and their Economic Benefits in Maintenance

tent type and quality (Papathanassiou et al., 2013). Relevant technological innovation brings greater flexibility, efficiency, mobility and ease of use due to specific functional design features, i.e. navigation patterns, short path to relevant knowledge, interface design patters, as well as content-related features that facilitate adoption by users. Additionally, regarding e-maintenance solutions adoption, one should appreciate efforts made in industry to reduce information and services fragmentation via appropriate integration mediation mechanisms, which remain technically transparent and not-noticeable by users, while, nonetheless, increasing the seamless experience of services usage and information delivery to maintenance personnel (Pistofidis et al., 2012).

\section{The economic benefits of using the mobile tech- nologies in Maintenance}

When addressing mobile technologies for maintenance, both business process analysis and the assessment of impacts must be conducted. Financial benefits that e-Maintenance technologies can offer must be addressed carefully, relying on the principles of the Cost Benefit Analysis (CBA). CBA is a well established methodology, see (Prest and Turvey 1965), and it is based on the evaluation of the possible costs and benefits of a project. Indeed, the CBA has been carried out by paying a specific attention to the fact that costs and benefits result from the introduction of new technologies. A methodology, previously developed for the assessment of new technologies in logistics, is one of the main and possible areas to exploit. Examples are provided by Miragliotta et al., (2009). The methodology consists of 3 steps. The first step is the Analysis of the AS-IS process, according to ABC (Activity Based Costing) method, in order to identify the cost drivers of each activity. The second step is the Development of a technical solution for the process under concern. Finally, the Assessment of the impacts of the solution and the evaluation of costs and benefits is carried out through a comparison between AS-IS and TOBE scenarios. In addition, Gilabert et al., (2015) presented how simulation tools can help identify a new predicting maintenance approach by means of ICTs in the cost-benefit of the product life cycle or plant productivity. The reliability information on which the maintenance strategies simulator relies is the probability density distribution of failure for the system or component. Such function determines the possibility of a failure occurring at a given time. The Weibull distribution is frequently employed because it is applicable to different phases in the life of a component or system. Given this function it is possible to apply the Monte Carlo method for performing a random sampling and as a consequence for obtaining possible times at which failure occurs. With this methodology it is possible to obtain a time of occurrence of a failure and, therefore, to anticipate the type and number of maintenance actions performed following a particular maintenance strategy and their result in terms of cost. 
${ }^{1}$ J.Campos - Mobile Technologies Acceptance and their Economic Benefits in Maintenance

This process is repeated according to the Monte Carlo method of analysis to offer a faithful description, and time and costs are accumulated. The cost per unit time is used in order to compare the results obtained with different maintenance strategies. Further on, while making the assessment of impacts, a classification of typical costs and benefits can be kept in mind. In particular, costs and benefits can be divided in Tangible and Intangible benefits (Fumagalli et al., 2010; Jantunen et al., 2010). A formal and clear understanding of the relation between the activities and the mobile technologies is a key aspect to be considered during the assessment. Fumagalli et al., (2010) demonstrated, with the help of a case study analysis, that significant benefits can be obtained with a small scale investment, whose payback time was (in 2010) reasonably well under three years in all the cases. Interestingly, 2010 can be considered still the time when mobile devices were not so diffused. Just to give an example, Apple iPad was presented in January 2010 and can be somehow considered the precursor of the tablets. Moreover, starting from 2010, the mass diffusion of this kind of portable devices increased. Of course, this type of mobile devices is not comparable with industrial mobile devices, but the diffusion of tablet as mass product has contributed to the cost reduction, allowing one to foresee that payback time could be within around only one year and maximum two years. Evidently, it depends on the type of company business and the way the company is organized. Fumagalli et al., (2010) showed that large companies offering a wide range of maintenance services to many different types of customers, performing their activities in a formal and procedure-driven way, can benefit from the newly introduced mobile technologies by gaining process efficiency. On the other hand, Original Equipment Manufacturers (OEM) with less developed maintenance division can benefit from efficiency, but this hardly compensates the costs of the introduction of mobile solution into the field service. To this end, it is always beneficial to consider developing the proper business model of maintenance service first, trying to avoid pushing it in terms of technology advantages.

\section{Industrial use of the mobile technologies}

In order to provide empirical evidence regarding the diffusion of using mobile solutions for maintenance, this section reports the research results obtained by the Observatory on Technologies and Services for Maintenance (TESEM, http://www.tesem.net/english-site), a permanent unit was created in December 2010 as part of the Observatories of the School of Management of Politecnico di Milano. TeSeM is a collaborative platform where a network of several Italian universities operates to monitor the state of the art on maintenance choices in the field of industrial plants. TeSeM observes small, medium and large companies in Italy. Indeed, TeSeM has collected information about the use of Personal Digital Assis- 
${ }^{1}$ J.Campos - Mobile Technologies Acceptance and their Economic Benefits in Maintenance

tant (PDA) and thus mobile solution for many multi-national European and worldwide companies, which are located in Italy. Information comes from 277 companies interviewed from 2011 to 2015 . Statistics show that $10 \%$ of the companies adopt such solutions. Moreover, considering the classification of smallmedium enterprises (SMEs) and big enterprises, the following statistics have been calculated according to the results of an interview conducted in the aforementioned companies. Specifically, in SMEs 6\% of companies adopt mobile solutions and in big enterprises $13 \%$ of the companies adopt mobile solutions. In more recent years, i.e. from 2013 to 2015, a more detailed analysis was performed for specific sectors. Results analysis reveals, for instance, that in the Food \& Beverages sector, $21 \%$ of companies adopt mobile solutions and the metalworking sector $5 \%$ of companies adopts mobile solutions. Finally, the end expectations regarding the use of mobile devices have been investigated. The results of the analysis are based on the number of companies that use mobile solutions, namely $10 \%$ of all the companies and show that in some cases companies use mobile solutions for more than one purpose. For instance, $18 \%$ of the companies that adopt mobile solutions use them for managing work orders, $2 \%$ exploit them for managing safety procedures of maintenance activities, $20 \%$ apply such technologies for supporting inspections, $18 \%$ for condition monitoring, 29\% for supporting spare parts warehouse management and $13 \%$ to consult technical documents and drawings.

\section{Discussion and Conclusions}

It is important to understand the best practices of Information Systems (IS) adoption as well as their different contributing factors in order to be able to facilitate the acceptance of ICTs and in particular mobile technologies in the maintenance departments. The importance is highlighted by the fact that e-Maintenance has created a radical change in maintenance practices. Consequently, having the correct information when is needed at hand can provide operational and financial benefits to most organisations. The advances in wireless technologies and low cost and easy to use mobile devices allow this to become a reality through portable, available and affordable systems. In addition, from an economical point of view, ICT-enabled innovation provides enormous opportunities. The most important functional benefit is the availability of relevant and reliable information and services where needed. On the other hand, efficient maintenance strategies such as CBM rely on information since they enable maintenance actions planning based on evidence of need and not merely on pre-determine schedules. Moreover, it is easy to understand how much support a maintenance engineer needs from an information system to carry out practical maintenance tasks. In all available economic studies the introduction of e-Maintenance has proved to be justified. Natu- 
${ }^{1}$ J.Campos - Mobile Technologies Acceptance and their Economic Benefits in Maintenance

rally, the more complicated the industrial environment is, the higher the economic benefits and the shorter the payback time for investments are expected to be.

\section{References}

Alrabghi A and Tiwari A., 2013. A Review of Simulation-based Optimisation in Maintenance Operations. 15th International Conference on Computer Modelling and Simulation (UKSim), pp. 353-358.

Baglee, D. Knowles.M., 2013. Maintenance Strategy Development in the UK Food and Drink Industry. International Journal of Strategic Engineering Asset Management, 1 (3), pp. $289-300$

Campos, J., 2009. Development in the application of ICT in condition monitoring and maintenance. Computers in Industry 60, pp. 1-20. doi:10.1016/j.compind.2008.09.007

Campos, J. \& Prakash, O., 2006. Information and communication technologies in condition monitoring and maintenance, in Dolgui, A., Morel, G and Pereira, C.E. (Eds.) In-formation Control Problems in Manufacturing. Elsevier.Vol. II, pp. 3-8.

Conde E, Fernandez S and Arnaiz A., 2009. Maintenance methodology for selecting the most cost-effective predictive strategies. 37th ESReDA Seminar 2009, Baden, Switzerland.

EFNMS/EAMC., 2012. How organizations manage their physical assets in practice, EFNMS Asset Management Survey (EFNMS).

Emmanouilidis, C, Liyanage, J.P. and Jantunen, E., 2009. Mobile solutions for engineering asset and maintenance management. J of Qual in Maintenance Eng 15 (1), pp. 92-105. doi:10.1108/13552510910943903

Fumagalli L., Di Leone F., Jantunen E., Macchi M., 2010. Economic Value of Technologies in an eMaintenance platform. (pp. 23- 28). In: 1st IFAC Workshop, A-MEST'10, Advanced Maintenance Engineering, Services and Technology. Lisboa, Portugal.

Gilabert, E., Fernandez, S., Arnaiz, A., Konde, E., 2015. Simulation of predictive maintenance strategies for cost-effectiveness analysis. Proceedings of the Institu- 
${ }^{1}$ J.Campos - Mobile Technologies Acceptance and their Economic Benefits in Maintenance

tion of Mechanical Engineers, Part B: Journal of Engineering Manufacture 0954405415578594. doi:10.1177/0954405415578594

Jantunen E., Macchi M., Fumagalli L., Di Leone F. 2010. Financial analysis of eMaintenance, MAINTWORLD, 2 pp. 38- 43

Lee, J, Ramji, A, Andrews K. , Darning L, Dragan, B., 2004. An integrated platform for diagnostics, prognostics and maintenance optimization, in e-Proceedings of Intelligent Maintenance System, Arles, France, 15-17 July

Miragliotta Giovanni, Alessandro Perego, Angela Tumino (2009). RFId and Returnable Transport Items Management: an Activity-Based Model to Assess the Costs and Benefits in the Fruit and Vegetable Supply Chain, Proceedings of the 3rd International Workshop on RFId Technology \& Concepts, Applications, Challenges, IWRT 2009, in conjunction with ICEIS 2009 (International Conference on Enterprise Information Systems), 2009, Milan, Italy.

Prest A. Richmond, R. Turvey. "Cost-Benefit Analysis: A Survey." The Economic Journal, 1965: 683-735.

Papathanassiou, N., Pistofidis, P., and Emmanouilidis, C.,2013. Competencies Development and Self-Assessment in Maintenance Management e-Training, European Journal of Engineering Education, v38(5), pp. 497-511, (Taylor \& Francis).

Pistofidis, P., Emmanouilidis, C., Koulamas, C., Karampatzakis, D., Papathanassiou, N., 2012. A Layered E-Maintenance Architecture Powered by Smart Wireless Monitoring Components, Proc. of the 2012 IEEE International Conference on Industrial Technology (ICIT 2012), pp. 390-395, Athens, Greece 19-21 March 2012, (IEEE).

Syafar, F., and Gao, J., (2013), Mobile collaboration technology in engineering asset maintenance: A Delphi study, Proc. of the 2013 IEEE 17th International Conference on Computer Supported Cooperative Work in Design (CSCWD), 27-29 June 2013, Whistler, BC, Canada, pp. 483 - 488 (IEEE).

Venkatesh, V., Morris, M.G., Davis, G.B., Davis, F.D., 2003. User Acceptance of Information Technology: Toward a Unified View. MIS Quarterly 27, pp. 425478 . 